\section{() OPEN ACCESS}

\title{
Guideline review: EASL clinical practice guidelines: drug-induced liver injury (DILI)
}

\author{
Paul N Brennan (D) , ,2 Peter Cartlidge, ${ }^{3}$ Thomas Manship, ${ }^{4}$ \\ John F Dillon (1) 3,5
}

\begin{abstract}
${ }^{1}$ Centre for Regenerative Medicine, The University of Edinburgh Edinburgh Medical School, Edinburgh, UK

${ }^{2}$ Molecular and Clinical Medicine, University of Dundee School of Medicine, Dundee, UK

${ }^{3}$ Department of Gastroenterology, Ninewells Hospital and Medical School, NHS Tayside, Dundee, UK ${ }^{4}$ Centre for Liver and Digestive Disorders, NHS Lothian, Edinburgh, UK

${ }^{5}$ Molecular and Clinical Medicine, University of Dundee College of Medicine Dentistry and Nursing, Dundee, UK
\end{abstract}

\section{Correspondence to} Dr Paul N Brennan, Centre for Regenerative Medicine, The University of Edinburgh Edinburgh Medical School, Edinburgh EH8 9YL, UK; p.z. brennan@dundee.ac.uk

Received 16 April 2021 Accepted 19 July 2021

\section{A Check for updates}

(c) Author(s) (or their employer(s)) 2021. Re-use permitted under CC BY-NC. No commercial reuse. See rights and permissions. Published by BMJ.

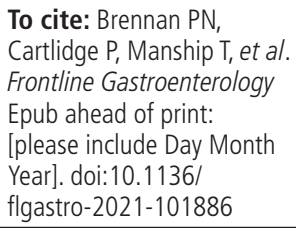

\begin{abstract}
The European Association for the Study of the Liver has produced extensive guidelines for the investigation and management of drug-induced liver injury. Here, we provide a commentary and overview of some of the principle disease investigations and management that arise from these guideline recommendations.
\end{abstract}

\section{OVERVIEW/COMMENTARY}

Guidelines have been produced by the European Association for the Study of the Liver for the investigation and management of drug-induced liver injury (DILI). DILI is epitomised by a myriad of presentations and idiosyncrasies, posing significant diagnostic challenge to generalists and hepatologists.

DILI is traditionally classified as intrinsic (or direct) versus idiosyncratic. Characteristically, intrinsic DILI is usually a predictable, dose-related phenomenon, occurring in a large subset of exposed individuals, with relatively short time to onset. Idiosyncratic DILI is not usually dose-related; however, a dose threshold is generally required, with variable latency of onset; from days to weeks. ${ }^{1}$

In addition to specific drug-related properties, there are important host predisposing factors including advancing age, sex, alcohol intake and underlying liver disease. Female sex appears to confer risk for DILI and have a higher risk of progression to acute liver failure. ${ }^{1}$ There are additional genetic drivers for DILI which vary widely and are covered extensively in the original guidelines.

A general approach to a suspected case of DILI is presented in figure 1. This includes taking a comprehensive medical and drug history, with clear timing around drug usage. Potential agents may include

\section{Key points}

- Drug-induced liver injury (DILI) can present with any recognised pattern of liver enzyme derangement.

- Diagnosis is complex, with no unifying criteria and a relatively high index of suspicion is necessary.

- Coexistent liver disease is an apparent risk factor for DILI.

- Standard assessment includes liver ultrasonography and screening for viral and autoimmune hepatitides.

- Histology may be useful in prognostication, particularly for those with slow to resolve disease or where there is diagnostic ambiguity.

- The mainstay of treatment is withdrawal of the offending agent.

- Clinicians may refer to LiverTox as a guide to recorded hepatotoxicity of specific agents. (https://www.ncbi.nlm.nih.gov/ books/NBK547852/).

both prescribed and non-prescribed compounds, herbal and dietary supplements (HDS), over-the-counter products and illicit substances.

HDS-associated liver toxicity appears to be an emerging determinant of DILI, with wide geographical variability. A prospective study in Iceland purported $16 \%$ of DILI related to HDS, ${ }^{2}$ while the US Drug-Induced Liver Injury Network reported a similar percentage $(16 \%)$, with an increase from $7 \%$ from 2004 to 2005 to $20 \%$ in $2013-2014 .^{3}$ Further casecontrol data suggested rates of $4 \%-5 \%$ in studies in Latin America ${ }^{4}$ and Germany, ${ }^{5}$ respectively.

DILI should be classified according to the dominant pattern of liver enzyme derangement; hepatocellular, cholestatic and mixed injury (figure 1). 


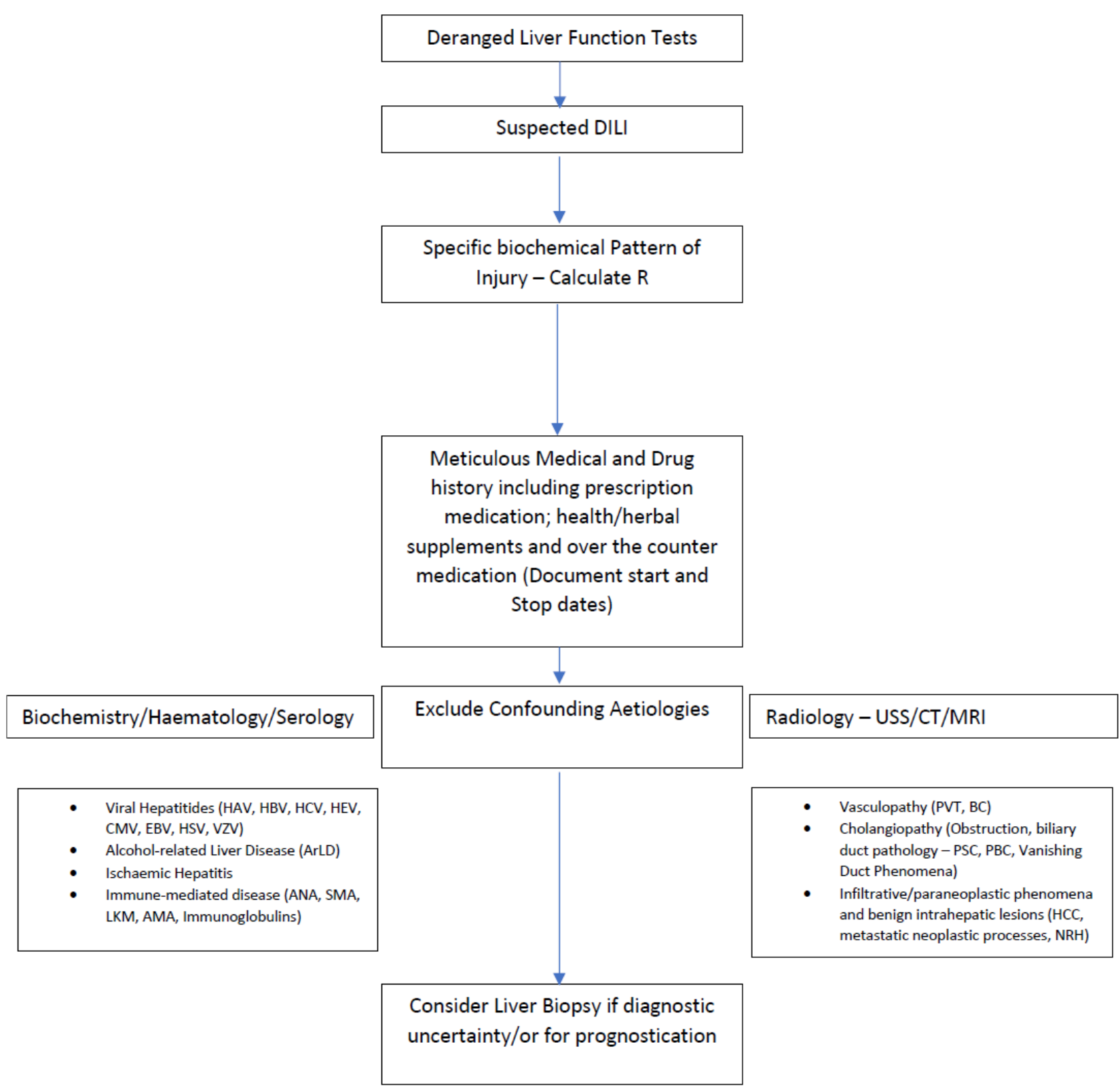

Figure 1 Suggested approach to presentation of drug-induced liver injury (DILI) ${ }^{1}$. ANA, antinuclear antibody; BC, Budd-Chiari syndrome; CMV, cytomegalovirus; EBV, Epstein-Barr virus; HAV, hepatitis A virus; HBV, hepatitis B virus; HCV, hepatitis C virus; HEV, hepatitis E virus; HSV, hepes simplex virus; HCC, hepatocellular carcinoma; LKM, liver microsomal antibody; NRH, nodular regenerative hyperplasia; PBC, primary biliary cholangitis; PSC, primary sclerosing cholangitis; PVT, portal vein thrombosis; SMA, smooth muscle antibody; USS, ultransound scan; VZV, varicella zoster virus.

Initially, alanine transferase (ALT) activity (patients ALT/upper limit of normal (ULN) of ALT) and alkaline phosphatase (ALP) activity (patients ALP/ULN of ALP) is calculated. Then ALT/ALP ratio (R) is determined. Some of the other commonly encountered phenotypes, and characteristic findings and commonly implicated agents are summarised in table 1.

ALT, ALP and bilirubin are the typical indices used to define liver damage, and liver dysfunction in DILI and serial measurements are necessary to portray extent of hepatocellular injury and DILI phase. ALT is sensitive for hepatocyte injury, particularly alongside an elevated bilirubin and this pattern is a reliable biomarker of liver injury in DILI. ${ }^{6}$ Elevated aminotransferases do not reflect the extent to which the liver is damaged, particularly in insidious variants of hepatotoxicity such as indolent fibrosis, vascular liver disorders, cirrhosis and microvesicular steatosis secondary to mitochondrial toxicity. Elevated ALP values usually indicate cholestatic damage, which combined with elevated GGT provides evidence the ALP elevation is of hepatic origin. Isolated hyperbilirubinaemia does not qualify as DILI, given possibility of multiple confounding diagnoses. One important consideration 
Table 1 Classification of DILI based on liver enzyme derangement ${ }^{1}$

\begin{tabular}{|c|c|c|}
\hline Phenotype & Case definition & Commonly implicated agents \\
\hline Idiosyncratic & $\begin{array}{l}\text { Hepatocellular: If ALT alone is elevated less than } \\
\text { fivefold above ULN or } R \geq 5 \text {. } \\
\text { Cholestatic: ALP alone is elevated less than twofold } \\
\text { above ULN or } R \leq 2 \text {. } \\
\text { Mixed: } R>2 \text { to }<5 \\
\text { Chronic DILI: DILI with acute presentation, with } \\
\text { evidence of persistent liver injury at }>1 \text { year after } \\
\text { its onset }\end{array}$ & $\begin{array}{l}\text { Antimicrobials, anticonvulsants, antiarrhythmic, } \\
\text { androgens, oestrogens/progesterone, immunomodulatory } \\
\text { and antineoplastic }\end{array}$ \\
\hline $\begin{array}{l}\text { Drug reaction with eosinophilia and systemic } \\
\text { symptoms }\end{array}$ & $\begin{array}{l}\text { Drug-related hypersensitivity with eosinophilia and } \\
\text { systemic inflammation }\end{array}$ & Anticonvulsants, NRTIs \\
\hline Drug-induced autoimmune hepatitis & $\begin{array}{l}\text { Acute DILI with serological and/or histological } \\
\text { features of AlH }\end{array}$ & NSAIDs, statins, minocycline and nitrofurantoin \\
\hline Secondary sclerosing cholangitis & $\begin{array}{l}\text { Presenting as acute DILI with histological/ } \\
\text { radiological features of sclerosing cholangiopathy }\end{array}$ & Inhalational anaesthetics, atorvastatin, 6-MP \\
\hline Granulomatous hepatitis & $\begin{array}{l}\text { Granulomas on histology with exposure to } \\
\text { implicated agent(s) }\end{array}$ & Anticonvulsants, sulphonamides \\
\hline Acute fatty liver & Acute development of microvesicular steatohepatitis & Reverse transcriptase inhibitors \\
\hline Drug-associated fatty liver disease & Consistent with NAFLD and attributable exposure & Methotrexate, corticosteroids, 5-FU \\
\hline Nodular regenerative hyperplasia & $\begin{array}{l}\text { Diffuse nodularity organised around central } \\
\text { hepatocytes }\end{array}$ & Antineoplastic/cytotoxic \\
\hline Ductopaenia & Chronic cholestasis and ductular loss & $\begin{array}{l}\text { Antimicrobials ( } \beta \text {-lactams, tetracyclines and } \\
\text { sulphonamides) }\end{array}$ \\
\hline Liver tumours & $\begin{array}{l}\text { Features of hepatocellular adenoma or carcinoma } \\
\text { dependent of histological/imaging characteristics }\end{array}$ & Anabolic androgenic steroids and oral contraceptives \\
\hline
\end{tabular}

5-FU, 5-fluorouracil; 6-MP, 6-mercaptopurine; AlH, autoimmune hepatitis; ALP, alkaline phosphatase; ALT, alanine transferase; DILI, drug-induced liver injury; NAFLD, non-alcoholic fatty liver disease; NRTI, nucleoside reverse transcriptase inhibitor; NSAID, non-steroidal antiinflammatory drug; ULN, upper limit of normal.

is mild transaminase rises that may be apparent in relation to statin therapy, this may simply reflect an adaptive response and does not represent a true DILI. As such, given the benefit of statins in NAFLD(Nonalcoholic fatty liver disease) and cardiovascular disease such therapies should be continued where feasible.

Patients with DILI should undergo testing for hepatotropic viruses including hepatitis (A-E), particularly in those with acute hepatocellular injury. For completeness an autoantibody screen (antinuclear antibody, anti-smooth muscle antibody (ASMA)), M2-anti-mitochondrial antibody (AMA), liver microsomal antibody, immunoglobulins) should be undertaken. DILI assessment should also include coagulation profiles, as elevated prothrombin time ratio values may suggest impending acute liver failure (ALF) and prompt referral to a liver transplant unit should be considered.

Abdominal ultrasound should be undertaken in all patients suspected of DILI to exclude any biliary, parenchymal or vasculopathy, additional imaging is dependent on the clinical context.

Liver biopsy may be reasonable to consider in DILI, as histology may provide information pertaining to severity of liver injury and provide mechanistic insights by identifying specific patterns of injury. Liver biopsy is also warranted in those patients suspected of DILI when serology raises the possibility of autoimmune hepatitis. ${ }^{1}$ Liver biopsy may also be considered in patients whereby suspected DILI progresses, or fails to resolve on withdrawal of the causal agent, since histology may provide prognostic information assisting clinical decision particularly regarding immunosuppression. ${ }^{7}$ There are characteristic histological patterns associated with individual check-point inhibitors including presence of ring granulomas and endotheliitis which may aid decision making around immunosuppression. ${ }^{1}$ In select cases of DILI, human leucocyte antigen genotyping can be used, whereby genetic determination may aid diagnosis and management, particularly those with features compatible with autoimmune hepatitis.

Given the non-specific nature of traditionally employed liver enzyme measurements there is increasing interest in determining novel serum biomarkers. These markers include glutamate dehydrogenase, keratin 18, glutathione S-transferase, sorbitol dehydrogenase, bile acids, cytochrome P450 and osteopontin. These markers may help to improve the specificity of DILI diagnosis, and aid prognostication. Presently, however, none are routinely employed, but represent exciting future avenues of research.

Rechallenging patients who had initial drugrelated liver injury can lead to rapid, progressive liver insult often worse than previous with fulminant hepatic failure. A positive rechallenge is defined as an ALT $>3$ ULN and is the strongest proof of drug causality. ${ }^{8}$ Some essential, irreplaceable medications may be used to rechallenge patients including; antituberculous and chemotherapy agents, however, this should only be considered with meticulous monitoring arrangements under specialist supervision. 
Another novel causative potentiator of DILI is those related to the classes of novel immunomodulatory therapeutic classes. These molecules are being increasingly used to treat malignancy through restitution of strong humoral, antitumour immune response, thereby improving patient survival. The reduction in tumour tolerance induced by immune checkpoint inhibitors can lead to inflammatory side effects, and an increase in immune related adverse events, including hepatotoxicity. Risk factors include the type of check point inhibitor, higher dose, autoimmune predisposition, pre-existing liver disease and the use of combination agents. ${ }^{9}{ }^{10}$ Hepatotoxicity is heterogeneous ranging from mild transaminase derangement to pronounced acute hepatitis and fulminant liver failure. Assessment of severity can be determined using Common Terminology Criteria for Adverse Events scale and persistent grade 2-4 hepatotoxicity, then immunosuppression should be considered, along with cessation of the causative agent. The American Gastoenterological Association (AGA) has produced a practice update relating to this including outline of treatment strategies including corticosteroids and steroid-sparing agents. ${ }^{11}$

The Council for International Organisations of Medical Sciences scale can be used to assess DILI causality and consists of seven domains. The score categorises cases as highly probable $(>8)$, probable $(6-8)$, possible (3-5), unlikely (1-2) or excluded $(<0)$ and is useful in initially evaluating likelihood of suspected DILI.

The principle management of suspected DILI is discontinuation of the likely causative agent. In the majority of DILI, spontaneous recovery occurs, without any need for treatment or specific supportive measures. This aspect of spontaneous recovery following discontinuation of an offending substance forms an important criterion in the causality assessment of DILI. There is no strong evidence for use of $\mathrm{N}$-acetyl cysteine) except in paracetamol and ALF. Of note, there is no role for corticosteroids beyond cases of presumed autoimmune hepatitis and immune-related adverse reactions or for the use of ursodeoxycholic acid in cholestatic DILI.

DILI remains a challenging presentation with specific diagnostic and treatment complexities. Notwithstanding this, there remain a number of outstanding aspects of disease pathogenesis and predisposing factors that necessitate further exploration. There also exists, a clear need for novel biomarkers and predictors of outcome, beyond existing genetic determinants. The advent of novel treatment modalities, particularly oncological immune modulators pose another challenging paradigm, and clinicians should be aware of their potential for hepatotoxicity.

One particularly useful resource which clinicians should be aware of is LiverTox. ${ }^{12}$ LiverTox provides contemporaneous, unbiased, and accessible reports on the diagnosis, cause, frequency, clinical patterns and management of liver injury attributable to prescription, non-prescription medications and selected HDS products.

Overall, however, given the inherent complexity of DILI, we would encourage clinicians to refer to the original guideline for points of reference as a definitive overview on the subject matter.

Twitter Paul N Brennan@brennap9

Contributors PNB reviewed the guideline and drafted initial manuscript. PC and TM reviewed the literature and prepared relevant sections and reviewed final document. JFD revised the manuscript critically for important intellectual content and provided critical appraisal.

Funding The authors have not declared a specific grant for this research from any funding agency in the public, commercial or not-for-profit sectors.

Competing interests JFD has received research grants and lecture honoraria from MSD, Abbvie and Gilead.

Patient consent for publication Not required.

Provenance and peer review Not commissioned; externally peer reviewed.

Open access This is an open access article distributed in accordance with the Creative Commons Attribution Non Commercial (CC BY-NC 4.0) license, which permits others to distribute, remix, adapt, build upon this work noncommercially, and license their derivative works on different terms, provided the original work is properly cited, appropriate credit is given, any changes made indicated, and the use is noncommercial. See: http://creativecommons.org/licenses/by-nc/4. $0 /$.

\section{ORCID iDs}

Paul N Brennan http://orcid.org/0000-0001-8368-1478

John F Dillon http://orcid.org/0000-0002-2164-4476

\section{REFERENCES}

1 European Association for the Study of the Liver. Electronic address: easloffice@easloffice.eu, Clinical Practice Guideline Panel: Chair:, Panel members, et al. EASL clinical practice guidelines: drug-induced liver injury. J Hepatol 2019;70:122261.

2 Björnsson ES, Bergmann OM, Björnsson HK, et al. Incidence, presentation, and outcomes in patients with drug-induced liver injury in the general population of Iceland. Gastroenterology 2013;144:1419-25.

3 Navarro VJ, Barnhart H, Bonkovsky HL, et al. Liver injury from herbals and dietary supplements in the U.S. drug-induced liver injury network. Hepatology 2014;60:1399-408.

4 Bessone F, García-Cortés M, Medina-Caliz I, et al. Herbal and dietary Supplements-Induced liver injury in Latin America: experience from the Latindili network. Clin Gastroenterol Hepatol 2021. doi:10.1016/j.cgh.2021.01.011. [Epub ahead of print: 09 Jan 2021].

5 Douros A, Bronder E, Andersohn F, et al. Herb-induced liver injury in the Berlin case-control surveillance study. Int J Mol Sci 2016;17. doi:10.3390/ijms17010114. [Epub ahead of print: 15 Jan 2016].

6 Senior JR. Can rechallenge be done safely after mild or moderate drug-induced liver injury? Hepatology 2016;63:691-3.

7 Kleiner DE, Brunt EM, Van Natta M, et al. Design and validation of a histological scoring system for nonalcoholic fatty liver disease. Hepatology 2005;41:1313-21.

8 Andrade RJ, Robles M, Lucena MI. Rechallenge in druginduced liver injury: the attractive hazard. Expert Opin Drug Saf 2009;8:709-14. 
9 Boutros C, Tarhini A, Routier E, et al. Safety profiles of antiCTLA-4 and anti-PD-1 antibodies alone and in combination. Nat Rev Clin Oncol 2016;13:473-86.

10 Wang W, Lie P, Guo M, et al. Risk of hepatotoxicity in cancer patients treated with immune checkpoint inhibitors: a systematic review and meta-analysis of published data. Int $J$ Cancer 2017;141:1018-28.
11 Dougan M, Wang Y, Rubio-Tapia A, et al. AGA clinical practice update on diagnosis and management of immune checkpoint inhibitor colitis and hepatitis: expert review. Gastroenterology 2021;160:j.gastro.2020.08.063:1384-93.

12 Bethesda (MD), National Institute of Diabetes and Digestive and Kidney Diseases;. LiverTox - NCBI Bookshelf. Available: https://www.ncbi.nlm.nih.gov/books/NBK547852/ 